\title{
Laser Stabilization on a Fiber Ring Resonator and Application to RF Filtering
}

\author{
Pierre-Henri Merrer, Olivier Llopis, and Gilles Cibiel
}

\begin{abstract}
The potential of optical fiber ring resonators for RF or microwave signals filtering on optical carriers is demonstrated on a short length high $Q$ resonator. The problem of the frequency shift due to the resonator self heating with the optical power is solved thanks to a Pound-Drever feedback loop. A multi frequency RF filter is obtained, with a frequency step of $205 \mathrm{MHz}$ between resonances, and a $3 \mathrm{~dB}$ bandwidth of $2.4 \mathrm{MHz}$. This corresponds to the computed optical resonator $3 \mathrm{~dB}$ bandwidth, and thus represents an efficient technique for the measurement of ultra high $Q$ optical resonators. In the field of microwave applications, the equivalent $Q$ factor obtained is particularly interesting in the upper microwave range.
\end{abstract}

Index Terms-High $Q$ optical resonator, fiber ring resonator, frequency locking, microwave filters, optical filters, microwaveoptical systems.

\section{INTRODUCTION}

$\mathrm{O}$ ptical resonators featuring very high quality factor $(Q)$, in the range of $10^{8}$ to $10^{10}$, will be involved in many applications both in the optical and microwave domains. These ultra high $Q$ values can be obtained using optical whispering gallery modes (WGM) resonators, such as silica spheres [1], fused micro-tores [2] or monocristalline polished disks [3]. These resonators combine both small size and extreme $Q$ factors. They may open a new technological field, if the difficulties towards their integration can be overcome. They may be particularly useful at microwave frequencies, where today no resonator featuring simultaneously a small size and a very high $Q$ factor is available. In this case, the RF (Radio Frequency) or microwave signal is transposed to the optical domain where it is filtered, then it is brought back to microwaves thanks to a fast photodiode.

However, resonator integration is not the only problem with these devices. One difficult problem is related to the sensitivity of the resonant frequency to the optical power. Because of the ultra high $Q$, any small amount of power induced in the resonator will change its frequency, thus causing the loss of the signal. This problem can be studied on a more conventional resonator, featuring a $Q$ factor in the

Manuscript received Mars 12, 2008. This work was supported by CNES and ANR (National Research Agency) funding.

P.H. Merrer and O. Llopis are with LAAS-CNRS, Toulouse University, France.

G. Cibiel is with CNES, Microwave and Time-Frequency Department, Toulouse, France.

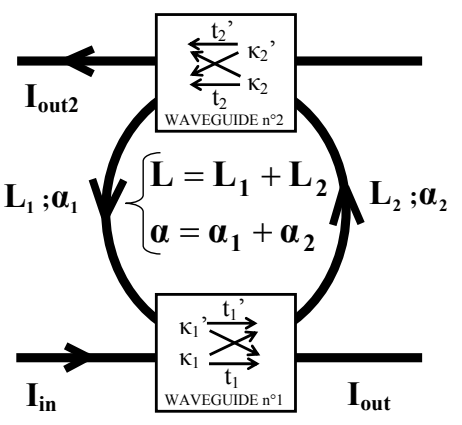

Fig. 1. Fiber ring resonator, with the transmission and coupling coefficients.

same range: the fiber ring resonator. With fiber ring resonators, the technological problem of resonator coupling is easily solved using low loss fiber couplers. The size of such a resonator is of course not as small as spheres or disks, but its planar geometry makes it potentially usable in many systems, including embedded systems. Nevertheless, like in WGM resonators, the sensitivity to the optical power level is strong in these resonators, and a special technique must be used to lock the resonator mode and the laser mode.

In this paper, we present the locking of a laser onto a fiber ring passive resonator at $1.5 \mu \mathrm{m}$ wavelength, featuring a $Q$ factor close to $10^{8}$. The locking is realized with a feedback on the laser using the Pound-Drever technique $[4,5]$. This technique has already been used in the case of micro-toroid resonators [6]. Our approach is however a bit different: we wanted to demonstrate the interest of the technique for RF signal stabilization, and to study the RF filtering potential of these resonators. We believe indeed that fiber ring resonators could be extensively used in optical microwave applications or for microwave generation in time and frequency applications. In a similar way, other authors have proposed, for these applications, a configuration involving an amplified self oscillating optical loop, featuring remarkable performances [7]. It is difficult to know at this time which of these two approaches will be more easily integrated in systems or which one will compete in terms of phase noise. However, the phase noise performance of these sources is already competitive with classical microwave frequency generation techniques.

Another interest in the approach presented here is that we propose an efficient and precise technique to measure the optical $Q$ factor of an ultra high $Q$ resonator, which otherwise is difficult to evaluate. This technique is based on a frequency domain approach, contrarily to the best known technique of "cavity ring down" [8], which is a time domain approach. 
TABLE I

MEASURED VALUES OF THE COUPLING AND LOSSES COEFFICIENTS

\begin{tabular}{cccc}
\hline \hline \multicolumn{2}{c}{ Waveguide $\mathrm{n}^{\circ} 1$} & \multicolumn{2}{c}{ Waveguide $\mathrm{n}^{\circ} 2$} \\
\hline $\mathrm{t}_{1}$ & $0,2 \mathrm{~dB}$ & $\mathrm{t}_{2}$ & $0,08 \mathrm{~dB}$ \\
$\mathrm{t}_{1}{ }^{\prime}$ & $0,19 \mathrm{~dB}$ & $\mathrm{t}_{2}{ }^{\prime}$ & $0,1 \mathrm{~dB}$ \\
$\kappa_{1}$ & $20,63 \mathrm{~dB}$ & $\kappa_{2}$ & $20,41 \mathrm{~dB}$ \\
$\kappa_{1}{ }^{\prime}$ & $20,47 \mathrm{~dB}$ & $\kappa_{2}$, & $20,26 \mathrm{~dB}$ \\
$\alpha_{1}$ & $0,01 \mathrm{~dB}$ & $\alpha_{2}$ & $0,03 \mathrm{~dB}$ \\
$\mathrm{~L}_{1}$ & $0,498 \mathrm{~m}$ & $\mathrm{~L}_{2}$ & $0,498 \mathrm{~m}$
\end{tabular}

\section{FIBER RING RESONATOR}

The optical resonator is represented in Fig. 1. It is formed by two waveguides coupled to single ring resonator realized with single mode telecommunication fiber. The resonant loop is fixed on a $10 \mathrm{~cm} \times 10 \mathrm{~cm}$ copper plate and embedded in a thermally isolated package. The coupling between the waveguides and the ring resonator can be described by transfer and scattering matrices. The response of this optical system is defined by the fiber loop length, L, by the coupling parameters $t, t^{\prime}, \kappa, \kappa^{\prime}$, and by the loop internal losses $\alpha$. This system allows to avoid problems related to coupling, and it has only two families of modes, TE (Transverse Electric) and TM (Transverse Magnetic) modes. The corresponding transfer function has been implemented on Matlab software, following an approach close to the one described in $[9,10]$. Table I depicts the actual coupling and losses parameters of this resonator. Fig. 2 shows, in a, the computed direct transmission through the first waveguide and, in $b$, the computed transmission through the second waveguide for the resonator we have realized. The optical quality factor, $Q_{\text {optic }}$ of the fiber ring resonator is computed from the half intensity spectral width $\Delta v_{F W H M}$ by the following relation:

$$
Q_{\text {optic }}=\frac{v}{\Delta \nu_{F W H M}}
$$

$v$ being the optical frequency. The theoretical analysis leads to $Q_{\text {optic }}=8.3 \times 10^{7}$ and the free spectral range $(F S R)$ of $205 \mathrm{MHz}$ with $\mathrm{L}=0.996 \mathrm{~m}$.

When this resonator is used for the filtering of a microwave signal, the spectral width of the filter is the same but the carrier frequency is reduced by the ratio between the optical and the microwave carriers. Thus an equivalent microwave $Q$ factor can be defined as:

$$
Q_{\text {microwave }}=Q_{\text {optic }} \frac{f}{v}
$$

$f$ being the microwave frequency. We therefore can get an equivalent $Q_{\text {microwave }}=8.3 \times 10^{3}$ at $20 \mathrm{GHz}$ with this ring resonator.

Up to now, microwave optical oscillators are realised with a long optical delay line (in the kilometre range) [11]. It is thus interesting to compare the equivalent $Q$ factor of the optical delay line $Q_{\text {delay line }}$ used in the optoelectronic oscillator (OEO) with the quality factor $Q_{\text {microwave }}$ of the fiber ring resonator. An equivalent $Q$ factor can be defined for a delay line by the following relation:

$$
Q_{\text {delay line }}=\pi f \tau
$$

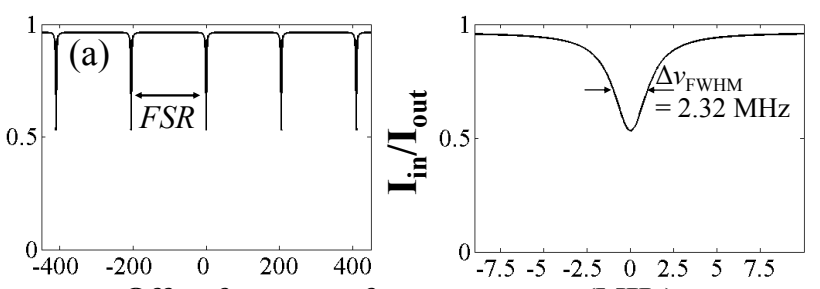

Offset frequency from resonance (MHz)

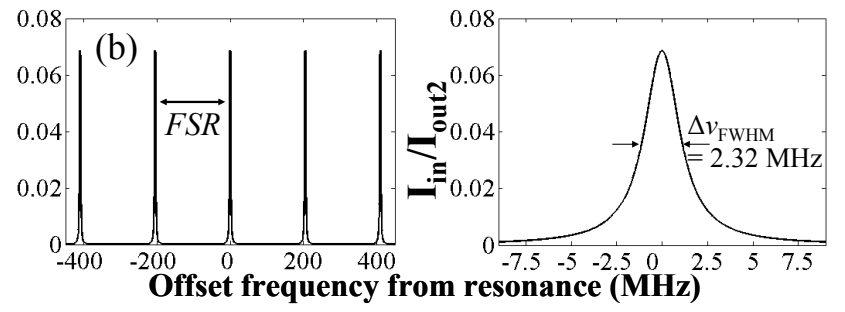

Fig. 2. Matlab computation of the normalized output intensity of the fiber ring resonator under test $\left(\mathrm{I}_{\text {out }}, \mathrm{I}_{\text {out } 2}\right)$ with measured values of the coupling and losses coefficients depicted in table I.

$\tau$ being the time delay of the signal after the delay line.

The improvement in $Q$ obtained using the resonator instead of delay line can be calculated by the following ratio R:

$$
\mathrm{R}=\frac{Q_{\text {microwave }}}{Q_{\text {delay line }}}=\frac{1}{\pi \cdot \tau \cdot \Delta \nu_{F W н M}}
$$

If we consider the special case of a fiber ring resonator illustrated by Fig. 1, an expression of $\Delta v_{F W H M}$ can be written according to coupling parameters and internal loss. From this expression, we obtain:

$$
\mathrm{R}=\frac{\sqrt{t_{1}^{\prime} t_{2} \alpha_{1} \alpha_{2}}}{\left(1-t_{1}^{\prime} t_{2} \alpha_{1} \alpha_{2}\right)}
$$

For the resonator we have realized, the improvement in $Q$ is:

$$
\mathrm{R}=\frac{Q_{\text {microwave }}}{Q_{\text {delay line }}}=28
$$

Thus to reach the same performance with the delay line technique, the fiber length should be increased by a factor 28 compared to the ring resonator, which is of course impossible in many applications. This factor increases even more (up to 90) if the internal losses in the ring resonator can be minimized.

\section{LASER AND FIBER RING RESONATOR LOCKED SYSTEM}

Using a resonator instead of a delay line is much more efficient but also more difficult from the system viewpoint. Indeed, the resonator only works at special frequencies, and thus need coherence between the laser frequency and the resonator frequency.

We use the Pound-Drever technique to stabilize the laser frequency by locking it to a resonance of the fiber ring resonator $[4,5]$. A schematic of the experimental setup is shown in Fig. 3. The laser used is a tunable fiber laser emitting at $\lambda=1550 \mathrm{~nm}$. Tunability is achieved by varying the temperature of the laser (dynamic range $=120 \mathrm{GHz}$ ), or by changing the voltage of a piezoelectric device (dynamic range $=3 \mathrm{GHz}$ ). The phase modulation is realized with a 


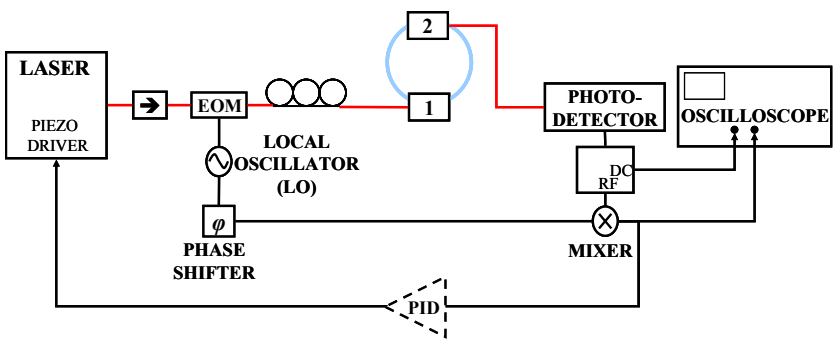

Fig. 3. Pound-Drever stabilization system.

$\mathrm{LiNbO}_{3}$ modulator. We also use an all-fiber polarization controller to get only one state of polarization, TE or TM. The Pound-Drever error signal is sent on a proportional integrator differentiator (PID). The correction is then applied to the piezoelectric device of the laser. Important factors affecting this loop are the controller gain and integration time. The oscillator frequency is set out at $40 \mathrm{MHz}$, which is a large value compared to the resonance width but smaller than the FSR.

\section{FIBER RING RESONATOR PERFORMANCE}

The optical fiber ring resonator can play the role of a high $Q$ microwave filter if it is used in a microwave link. For applications purpose it is interesting to measure the quality factor $Q$ of such a resonator. This technique is also of interest to determine the optical $Q$ factor of very high $Q$ resonators, even if no specific RF or microwave application is foreseen.

The measure of the resonator quality factor $Q$ requires intensity modulation of the laser beam. This is obtained by using a Mach-Zehnder electro-optic modulator which creates lateral sidebands around the optical carrier. The optical carrier must initially be locked to a cavity resonance by using the Pound-Drever method. The two sidebands go through the two lateral modes of the resonator (one FSR away from the laser frequency), and are thus filtered by these modes. This filtering effect, observed between the modulation signal (MachZehnder input) and the photodiode output, is measured using an RF network analyser. Fig. 4 shows this measurement for different settings of the polarization controller. When only one state of polarization can be obtained, an optical quality factor of $Q=8.0 \times 10^{7}$ is measured, together with an FSR of $205 \mathrm{MHz}$. This measurement agrees very well with the theoretical value of $8.3 \times 10^{7}$ computed for the $Q$ factor of this resonator. If we consider the use of this resonator in a microwave application, lets say at $20 \mathrm{GHz}$, the $Q$ factor is $Q_{\text {microwave }}=8.3 \times 10^{3}$ which is about 4 to 8 times the loaded $Q$ factor value of a microwave dielectric resonator at the same frequency [12], and much higher $Q$ factors can be reached using longer loops, which makes this approach competitive compared to conventional microwaves approaches.

\section{V.CONCLUSION}

The use of a fiber ring resonator, together with PoundDrever laser stabilization approach, has demonstrated great potentialities for the filtering of RF signals on optical carriers.

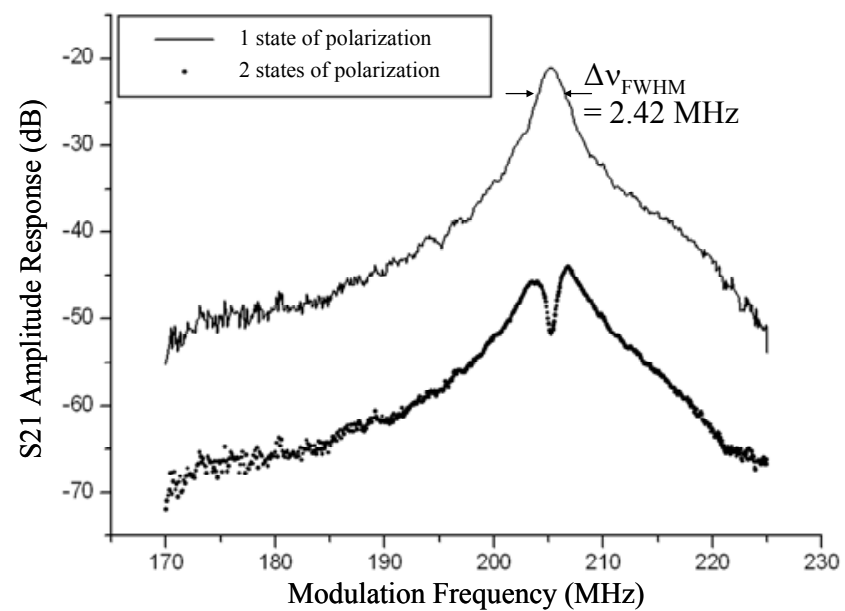

Fig. 4. Measured frequency responses of the fiber ring resonator for different settings of the polarization controller when the laser is locked onto a resonance. The measured full width at half maximum of $\Delta v_{\mathrm{FWHM}}=2.42 \mathrm{MHz}$ corresponds to an optical quality factor of $Q=8.0 \times 10^{7}$.

This technique opens the way to ultra high $Q$ microwave filtering and oscillator stabilization. As the equivalent RF or microwave $Q$ factor obtained is independent of the RF frequency, these systems may induce a real technological breakthrough in the high microwave range, with expected performances much higher than classical microwave systems.

\section{REFERENCES}

[1] M.L. Gorodetsky, A.A. savchenkov, and V.S. Ilchenko, "Ultimate Q of optical microsphere resonators," Opt. Letters, vol. 21, no. 7, pp. 453455, Apr. 1996

[2] D. K. Armani, T.J. Kippenberg, S.M. Spillane and K.J. Vahala, "Ultrahigh- $Q$ toroid microcavity on a chip," Nature, vol. 421, pp. 925-928, Feb. 2003.

[3] A.A. Savchenkov, V.S. Ilchenko, A.B. Matsko, and L. Maleki, "Kilohertz optical resonances in dielectric crystal cavities," Phys. Rev. A, vol.70, Issue 5, Nov. 2004.

[4] R. V. Pound, "Electronic Frequency Stabilization of Microwave Oscillators," Rev. Sci. Instrum., vol.17, no.11, pp.490-505, Nov. 1946.

[5] R. W. P. Drever, J. L. Hall, F. V. Kowalski et al., "Laser Phase and Frequency Stabilization Using an Optical-Resonator," Appl. Phys. B., vol. 31, no. 2, pp. 97-105, Jun. 1983.

[6] T. Carmon, T. Kippenberg, L. Yang, H. Rokhsari, S. Spillane, and K. Vahala, "Feedback control of ultra-high-Q microcavities: application to micro-Raman lasers and microparametric oscillators," Opt. Express, vol. 13, Issue 9, pp. 3558-3566, May 2005.

[7] D. Eliyahu, L. Maleki, "Modulation response (S21) of the coupled optoelectronic oscillator," Proc. of the IEEE IFCS 2005, pp. 850-856, Aug. 2005.

[8] A.A. Savchenkov, A.B. Matsko, M. Mohageg, and L. Maleki, "Ringdown spectroscopy of stimulated Raman scattering in a whispering gallery mode resonator," Opt. Letters, vol. 32, no. 5, pp. 497-499, Mar. 2007.

[9] A. Yariv, "Critical coupling and its control in optical waveguide-ring resonator systems," IEEE Photon. Technol. Lett., vol. 14, no.4, pp. 483485, Apr. 2002.

[10] A. Yariv, "Universal relations for coupling of optical power between microresonators and dielectric waveguides," Electron. Lett., vol.36, p.321-322, Feb. 2000

[11] X.S. Yao, L. Maleki, "Progress in the optoelectronic oscillator - a ten year anniversary review”, IEEE MTT-S 2004, vol. 1, pp. 287-290, Jun. 2004.

[12] B. Onillon, J. Rayssac, O. Llopis, B. Benazet, "Optimized Optical Links for High Spectral Purity Ku-band Signal Distribution", Proc. of the IEEE IFCS 2006, pp. 513-518, Aug. 2005. 